EXPLICIT NONFLAT TIME EVOLUTION FOR

PDE-BASED IMAGE RESTORATION

By

Seongjai Kim

and

Song-Hwa Kwon

IMA Preprint Series \# 2129

( July 2006)

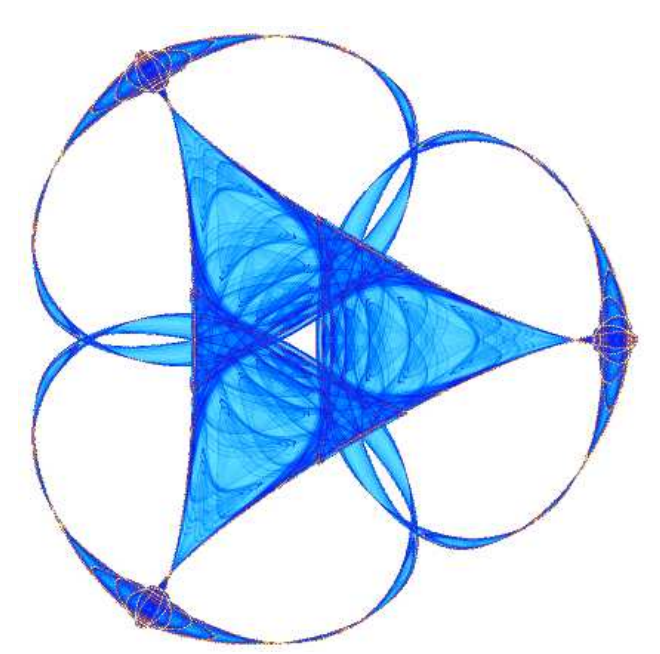

INSTITUTE FOR MATHEMATICS AND ITS APPLICATIONS

UNIVERSITY OF MINNESOTA 400 Lind Hall

207 Church Street S.E.

Minneapolis, Minnesota 55455-0436

Phone: 612-624-6066 Fax: 612-626-7370

URL: http://www.ima.umn.edu 


\title{
Explicit Nonflat Time Evolution for PDE-based Image Restoration
}

\author{
Seongjai Kim and Song-Hwa Kwon \\ Department of Mathematics \& Statistics, Mississippi State University \\ Mississippi State, MS 39762 USA Email: skim@math.msstate.edu \\ IMA, University of Minnesota, 207 Church Street S.E. \\ Minneapolis, MN 55455 Email: shkwon@ima.umn.edu
}

\begin{abstract}
This article is concerned with new strategies with which explicit time-stepping procedures of PDE-based restoration models converge with a similar efficiency to implicit algorithms. Conventional explicit algorithms often require hundreds of iterations to converge. In order to overcome the difficulty and to further improve image quality, the article introduces new spatially variable constraint term and timestep size, as a method of nonflat time evolution (MONTE). It has been verified that the explicit time-stepping scheme incorporating MONTE converges in only 4-15 iterations for all restoration examples we have tested. It has proved more effective than the additive operator splitting (AOS) method in both computation time and image quality (measured in PSNR), for most cases. Since the explicit MONTE procedure is efficient in computer memory, requiring only twice the image size, it can be applied particularly for huge data sets with a great efficiency in computer memory as well.
\end{abstract}

\section{Introduction}

Partial differential equation (PDE)-based image processing has been a popular tool for image restoration, since the first anisotropic diffusion model by Perona and Malik in 1990 [1]. A considerable amount of research has been carried out for the theoretical and computational understanding of various models; see e.g., [2-9] and [10-12]. It is now well understood that by choosing proper energy functionals in variational formulation and scaling their stationary EulerLagrange equations by appropriate factors, the resulting evolutionary models can restore important image features relatively well.

However, most of conventional PDE-based restoration models tend either to converge to a piecewise constant image or to lose fine structures of the given image, particularly unless they are both incorporating appropriate parameters and discretized by suitable numerical schemes. Although these results are important for understanding the current diffusion-like models, the resultant signals may not be desired in applications where the preservation of both slow transitions and fine structures is important. More advanced models are yet to be developed, along with effective strategies for the choice of appropriate parameters and numerical schemes. 
It is often the case that conventional explicit algorithms for solving PDEbased restoration models require hundreds of iterations to converge. This article introduces numerical strategies for the selection of spatially variable constraint parameter and timestep size, as a method of nonflat time evolution (MONTE), with which explicit procedures can converge fast and restore images in a comparable quality with implicit algorithms. In MONTE, the constraint parameter is selected larger on fast transitions, which in turn can suppress undesired dissipation effectively there; the timestep size is set reversely proportional to the sum of the diffusion coefficient and the constraint parameter, which makes the explicit procedure stable. Note that the MONTE solution must be defined on a nonflat time surface.

The new strategies have been implemented, incorporating an anisotropic diffusion spatial scheme, for both additive operator splitting (AOS) method [13] and the explicit algorithm. The explicit algorithm incorporating MONTE turns out to converge in 4-15 iterations for all restoration examples we have tested. Furthermore, it has often restored better images (measured in PSNR) than the AOS algorithm. Since the new explicit MONTE procedure is efficient in computer memory, requiring only twice the image size, it can be applied, particularly for huge data sets (e.g., 3D images), with a great efficiency in both convergence and computer memory.

An outline of the paper is as follows. In the next section, we briefly review PDE-based restoration models and their linearized time-stepping methods, followed by an anisotropic diffusion spatial scheme. Section 3 contains new numerical strategies for variable constraint parameters, variable timestep sizes, and their applications to the explicit time-stepping method. In Section 4, we present numerical results to show efficiency (in computation time) and effectiveness (in the preservation of important image features) of the explicit MONTE procedure. Section 5 conclude our developments and experiments.

\section{Preliminaries}

In this section, we review briefly PDE-based restoration models and their linearized time-stepping procedures, followed by an anisotropic diffusion spatial scheme.

\subsection{PDE-based restoration models}

Let $u_{0}$ be an observed image of the form

$$
u_{0}=u+v,
$$

where $u$ is the desired image and $v$ denotes a mean-zero noise of variance $\sigma^{2}$. Then, popular PDE-based restoration models can be written in the following general form:

$$
\frac{\partial u}{\partial t}+S(u)=R\left(u_{0}-u\right),
$$

where $S$ is a (nonlinear) diffusion operator and $R$ denotes a nonnegative constraint term. For example, the Perona-Malik (PM) model [1], the total variation 
(TV) model [8], the improved TV (ITV) model [6,11], and the convex-concave anisotropic diffusion (CCAD) model $[4,5]$ can be specified as follows:

$$
\begin{aligned}
& S(u)=-\nabla \cdot(c(|\nabla u|) \nabla u), R=0, \quad(\mathrm{PM}) \\
& S(u)=-\kappa_{1}(u), \quad R=\lambda, \quad(\mathrm{TV}) \\
& S(u)=-|\nabla u| \kappa_{1}(u), \quad R=\lambda|\nabla u|, \quad(\text { ITV }) \\
& S(u)=-|\nabla u|^{q} \kappa_{q}(u), \quad R=\beta\left|u_{0}-u\right|,(\mathrm{CCAD})
\end{aligned}
$$

where $\lambda, \beta \geq 0,0 \leq q<2$, and

$$
c(x)=\left(1+x^{2} / K^{2}\right)^{-1}, \quad \kappa_{q}(u)=\nabla \cdot\left(\frac{\nabla u}{|\nabla u|^{q}}\right),
$$

for some $K>0$.

The PM and TV models tend to converge to a piecewise constant image; such a phenomenon is called the staircasing effect. To suppress the staircasing effect, Marquina and Osher [6] suggested the ITV model, a scaling of the TV model by a factor of $|\nabla u|$. Since $|\nabla u|$ vanishes only on flat regions, its steady state is analytically the same as that of the TV model. The ITV model turns out to reduce the staircasing effect successfully; however, it is yet to be improved for a better preservation of fine structures. The CCAD model is a non-variational generalization of the ITV model and can be implemented as a stable numerical algorithm for $q \geq 0$; see [5] for details.

Note that the image is originally time-independent; the time in (2) has been introduced in order to deal with the corresponding steady-state PDEs conveniently. Thus the time is an artificial variable and can be considered as an algorithmic parameter for the solution, the restored image.

\subsection{Linearized time-stepping procedures}

Let $\Delta t^{n}$ be the $n$th timestep size and $t^{n}=\sum_{i=1}^{n} \Delta t^{i}, n \geq 1$, with $t^{0}=0$. Define $u^{n}=u\left(\cdot, t^{n}\right), n \geq 0$, with $u^{0}=u_{0}$. Given $u^{0}, \cdots, u^{n-1}$, we will try to compute $u^{n}$ by linearized time-stepping procedures. For $\ell=1,2$, let $\mathcal{S}_{\ell}^{n-1}$ be diffusion matrices approximating directional operators of the diffusion term $S$; for example, for the CCAD model,

$$
\mathcal{S}_{\ell}^{n-1} u^{m} \approx-\left|\nabla u^{n-1}\right|^{q} \partial_{x_{\ell}}\left(\frac{\partial_{x_{\ell}} u^{m}}{\left|\nabla u^{n-1}\right|^{q}}\right), \quad m=n-1, n .
$$

(See Section 2.3 below for details of an anisotropic diffusion spatial scheme.) Define $\mathcal{A}^{n-1}=\mathcal{A}_{1}^{n-1}+\mathcal{A}_{2}^{n-1}$, where

$$
\mathcal{A}_{\ell}^{n-1}=\mathcal{S}_{\ell}^{n-1}+\frac{1}{2} \mathcal{R}^{n}, \quad \ell=1,2 .
$$

Here $\mathcal{R}^{n}$ is an evaluation of the constraint term $R$ for the $n$th time level, of which an effective strategy will be considered in Section 3.1. Then, a linearized $\theta$-method for (2) can be formulated as follows: for $0 \leq \theta \leq 1$,

$$
\left.\frac{u^{n}-u^{n-1}}{\Delta t^{n}}+\mathcal{A}^{n-1}\left[\theta u^{n}+(1-\theta) u^{n-1}\right)\right]=\mathcal{R}^{n} u_{0} .
$$


For $\theta=0$, the $\theta$-method computes $u^{n}$ explicitly; when $\Delta t^{n}$ is constant spatially, it must be sufficiently small in order for the algorithm to be stable. On the other hand, for $\theta>0$, one can solve the linear system (6) by applying an iterative algebraic solver or the alternating direction implicit (ADI) procedure $[14,15,4]$.

Although the algebraic system (6) is often solved implicitly $(\theta=1 / 2$ or 1$)$ in the literature, the explicit procedure $(\theta=0)$ is still popular, due to simplicity in implementation and efficiency in computer memory. However, it requires to choose $\Delta t^{n}$ sufficiently small for stability and therefore converges in a huge number of iterations, which is its major disadvantage. In Section 3.2, we will study a strategy for the choice of spatially variable $\Delta t^{n}$, i.e., $\Delta t^{n}=\Delta t^{n}(\mathbf{x})$; with which the explicit procedure can converge quickly, e.g., in about 10 iterations. In Section 4, the new explicit algorithm will be compared with the AOS method:

$$
\begin{aligned}
& \frac{u^{n, k}-u^{n-1}}{\Delta t^{n}}+2 \mathcal{A}_{k}^{n-1} u^{n, k}=\mathcal{R}^{n} u_{0}, \quad k=1,2, \\
& u^{n}=\left(u^{n, 1}+u^{n, 2}\right) / 2,
\end{aligned}
$$

which holds the maximum principle independently of the timestep size and involves a splitting error of $\mathcal{O}(\Delta t)$; see [13].

\subsection{An anisotropic diffusion spatial scheme}

For a completeness of the article, this subsection presents an anisotropic diffusion scheme for $\mathcal{S}_{\ell}^{n-1}$ utilized in (4), which was first introduced in [16]. We will show the construction of $\mathcal{S}_{1}^{n-1}$; the analogue can be applied to obtain $\mathcal{S}_{2}^{n-1}$. Let $\mathcal{D} u_{i-1 / 2, j}^{n-1}$ be a finite difference approximation of $\left|\nabla u^{n-1}\right|$ evaluated at $\mathbf{x}_{i-1 / 2, j}$, the mid point of $\mathbf{x}_{i-1, j}$ and $\mathbf{x}_{i, j}$. For example, a second-order scheme reads

$$
\begin{aligned}
\mathcal{D} & u_{i-1 / 2, j}^{n-1}=\left(\left(u_{i, j}^{n-1}-u_{i-1, j}^{n-1}\right)^{2}\right. \\
& \left.+\left[\frac{1}{2}\left(\frac{u_{i-1, j+1}^{n-1}+u_{i, j+1}^{n-1}}{2}-\frac{u_{i-1, j-1}^{n-1}+u_{i, j-1}^{n-1}}{2}\right)\right]^{2}\right)^{1 / 2} .
\end{aligned}
$$

Define

$$
d_{i j, W}^{n-1}=\left[\left(\mathcal{D} u_{i-1 / 2, j}^{n-1}\right)^{2}+\varepsilon^{2}\right]^{q / 2}, \quad d_{i j, E}^{n-1}=d_{i+1, j, W}^{n-1},
$$

where $\varepsilon$ is a positive constant (small) introduced to prevent $d_{i j, W}^{n-1}$ from approaching zero. Then the differential operators in $(4), \ell=1$, can be approximated as

$$
\begin{aligned}
& -\partial_{x_{1}}\left(\frac{\partial_{x_{1}} u^{m}}{\left|\nabla u^{n-1}\right| q}\right) \approx-\frac{1}{d_{i j, W}^{n-1}} u_{i-1, j}^{m} \\
& +\left(\frac{1}{d_{i j, W}^{n-1}}+\frac{1}{d_{i j, E}^{n-1}}\right) u_{i, j}^{m}-\frac{1}{d_{i j, E}^{n-1}} u_{i+1, j}^{m}, \\
& \left|\nabla u^{n-1}\right|^{q} \approx \frac{2 d_{i j, W}^{n-1} \cdot d_{i j, E}^{n-1}}{d_{i j, W}^{n-1}+d_{i j, E}^{n-1}} .
\end{aligned}
$$

Note that the last approximation is the harmonic average of $d_{i j, W}^{n-1}$ and $d_{i j, E}^{n-1}$ and first-order accurate. It follows from (4) and (10) that the three consecutive 
non-zero elements of the matrix $\mathcal{S}_{1}^{n-1}$ corresponding to the pixel $\mathbf{x}_{i j}$ read

$$
\left[\mathcal{S}_{1}^{n-1}\right]_{i j}=\left(-s_{i j, W}^{n-1}, 2,-s_{i j, E}^{n-1}\right),
$$

where

$$
s_{i j, W}^{n-1}=\frac{2 d_{i j, E}^{n-1}}{d_{i j, W}^{n-1}+d_{i j, E}^{n-1}}, \quad s_{i j, E}^{n-1}=\frac{2 d_{i j, W}^{n-1}}{d_{i j, W}^{n-1}+d_{i j, E}^{n-1}} .
$$

Note that $s_{i j . W}^{n-1}+s_{i j . E}^{n-1}=2$. The above non-standard, anisotropic diffusion numerical scheme has been successfully applied for image zooming of arbitrary magnification factors $[17,16]$ and a simultaneous denoising and edge enhancement [5].

\section{The Method of Nonflat Time Evolution (MONTE)}

In this section, we will introduce an effective variable constraint parameter $\mathcal{R}^{n}=\mathcal{R}^{n}(\mathrm{x})$, followed by an explicit scheme incorporating variable timestep size $\Delta t^{n}=\Delta t^{n}(\mathbf{x})$.

\subsection{Constraint parameters}

For most PDE-based models, the constraint parameter has been chosen as constant, due to simplicity. However, constant constraint parameters can often be ineffective in the preservation of interesting image features such as edges and textures, because the diffusion operator may introduce an extra dissipation on fast transitions.

In order to overcome the difficulty, one consider a variable constraint parameter as follows: Multiply the stationary part of $(2)$ by $\left(u_{0}-u\right)$ and average the resulting equation locally to obtain

$$
R(\mathbf{x}) \approx \frac{1}{\sigma_{\mathbf{x}}^{2}} \frac{1}{\left|\Omega_{\mathbf{x}}\right|} \int_{\Omega_{\mathbf{x}}}\left(u_{0}-u\right) S(u) d \mathbf{x},
$$

where $\Omega_{\mathbf{x}}$ is a neighborhood of $\mathbf{x}$ (e.g., the window of $(3 \times 3)$ pixels centered at $\mathbf{x})$ and $\sigma_{\mathbf{x}}^{2}$ denotes the local noise variance measured over $\Omega_{\mathbf{x}}$. Then, the right side of the above equation can be approximated as

$$
R(\mathbf{x}) \approx \frac{1}{\sigma_{\mathbf{x}}^{2}}\left\|u_{0}-u\right\|_{\mathbf{x}} \cdot\|S(u)\|_{\mathbf{x}}
$$

where $\|g\|_{\mathbf{x}}$ denotes a local average $|g|$ over $\Omega_{\mathbf{x}}$. The constraint parameter in (13) is proportional to both the absolute residual $\left|u_{0}-u\right|$ and the diffusion magnitude $|S(u)|$, which may effectively suppress the extra dissipation arising on fast transitions. Note that the local noise variance $\sigma_{\mathbf{x}}^{2}$ must be estimated appropriately; see [18] for an effective estimation of $\sigma_{\mathbf{x}}^{2}$.

Let the neighborhood $\Omega_{\mathbf{x}}$ be chosen to include a single pixel $\mathbf{x}$. Then the constraint parameter in (13) related to a pixel $(i, j)$ in the $n$th time level can be formulated as

$$
R_{i j}^{n}=\eta_{1} \cdot\left|u_{0, i j}-u_{i j}^{n-1}\right| \cdot\left|\left(\mathcal{S}^{n-1} u^{n-1}\right)_{i j}\right|,
$$

where $\eta_{1}$ is nonnegative constant and $\mathcal{S}^{n-1}=\mathcal{S}_{1}^{n-1}+\mathcal{S}_{2}^{n-1}$. 


\subsection{An explicit nonflat time-stepping procedure}

For $\theta=0$, the $\theta$-method (6) can be rewritten as

$$
u^{n}=\left(1-\Delta t^{n} \mathcal{S}^{n-1}\right) u^{n-1}+\Delta t^{n} \mathcal{R}^{n}\left(u_{0}-u^{n-1}\right) .
$$

Let the diffusion matrix $\mathcal{S}^{n-1}$ incorporate a five-point stencil, i.e.,

$$
\begin{aligned}
& {\left[\mathcal{S}^{n-1} u^{n-1}\right]_{i j}=s_{i j, C}^{n-1} u_{i j}^{n-1}-s_{i j, W}^{n-1} u_{i-1, j}^{n-1}} \\
& \quad-s_{i j, E}^{n-1} u_{i+1, j}^{n-1}-s_{i j, S}^{n-1} u_{i, j-1}^{n-1}-s_{i j, N}^{n-1} u_{i, j+1}^{n-1},
\end{aligned}
$$

where $s_{i j, W}^{n-1}, s_{i j, E}^{n-1}, s_{i j, S}^{n-1}, s_{i j, N}^{n-1} \geq 0$ and $s_{i j, C}^{n-1}:=s_{i j, W}^{n-1}+s_{i j, E}^{n-1}+s_{i j, S}^{n-1}+s_{i j, N}^{n-1}$. For the diffusion matrix $\mathcal{S}_{1}^{n-1}$ in $(11)$ and its analogue $\mathcal{S}_{2}^{n-1}$, we have $s_{i j, C}^{n-1}=4$.

When (16) and (14) are adopted respectively for the diffusion and constraint terms, the explicit procedure (15) can be written as

$$
\begin{aligned}
u_{i j}^{n}= & {\left[1-\Delta t^{n}\left(s_{i j, C}^{n-1}+R_{i j}^{n}\right)\right] u_{i j}^{n-1} } \\
& +\Delta t^{n}\left(s_{i j, W}^{n-1} u_{i-1, j}^{n-1}+s_{i j, E}^{n-1} u_{i+1, j}^{n-1}\right. \\
& \left.+s_{i j, S}^{n-1} u_{i, j-1}^{n-1}+s_{i j, N}^{n-1} u_{i, j+1}^{n-1}\right)+\Delta t^{n} R_{i j}^{n} u_{0, i j} .
\end{aligned}
$$

The above iteration is stable when all coefficients in the right side are nonnegative. Thus the stability condition for (17) reads

$$
\Delta t^{n} \leq \frac{1}{s_{i j, C}^{n-1}+R_{i j}^{n}} .
$$

A common practice for the choice of $\Delta t^{n}$ is

$$
\Delta t^{n}=\min _{i j} \frac{1}{s_{i j, C}^{n-1}+R_{i j}^{n}} .
$$

Recall that $R_{i j}^{n}$ is proportional to both the absolute residual and the diffusion magnitude, while $s_{i j, C}^{n-1}$ is a multiple of the diffusion coefficient. Thus the timestep size $\Delta t^{n}$ in (19) has been chosen as a constant, in order for the algorithm (17) not to introduce nonphysical oscillations (instability) on regions of the fastest transition. However, this choice can slow down evolution of the solution on other regions, particularly on slow transitions. This is why conventional explicit methods have often required hundreds of iterations to converge.

As an alternative to (19), this article considers the following variable timestep size $\Delta t^{n}=\Delta t^{n}(\mathbf{x})$ :

$$
\Delta t_{i j}^{n}=\frac{1}{s_{i j, C}^{n-1}+R_{i j}^{n}} .
$$

The above choice of a variable timestep size deserves the following remarks:

- On slow transitions, the constraint parameter $R_{i j}^{n}$ approaches zero and therefore a larger timestep must be set, which in turn makes the algorithm work faster in image restoration. 
- Since PDE-based models often incorporate an extra (faster) diffusion on fast transitions, the choice in (20) can serve as a modulator which tries to equalize the speed of diffusion over the image domain.

- The computed solution $u^{n}$ resides on a nonflat time surface, which causes no difficulties. Note that the time in PDE-based denoising models of interests has been introduced, as an artificial variable, in order to enhance convenience in numerical simulation. The variable timestep size in (20) can be viewed as a variable parameter of the algorithm (15) which is introduced to solve steady-state problems of the form

$$
S(u)=R\left(u_{0}-u\right) .
$$

Here we have chosen $\Delta t^{n}$ to enhance efficiency in algorithmic convergence. We will see in Section 4 that the choice is also effective in quality of image restoration.

- With (20), the algorithm (17) can be rewritten as

$$
\begin{aligned}
u_{i j}^{n}= & \frac{1}{s_{i j, C}^{n-1}+R_{i j}^{n}}\left(s_{i j, W}^{n-1} u_{i-1, j}^{n-1}+s_{i j, E}^{n-1} u_{i+1, j}^{n-1}\right. \\
& \left.+s_{i j, S}^{n-1} u_{i, j-1}^{n-1}+s_{i j, N}^{n-1} u_{i, j+1}^{n-1}+R_{i j}^{n} u_{0, i j}\right),
\end{aligned}
$$

which is an average of $u_{0, i j}$ and four neighboring pixel values of $u^{n-1}$; the weights are computed anisotropically, incorporating all the pixel values of $u^{n-1}$ on the $(3 \times 3)$ window centered at $(i, j)$, as presented in Section 2.3.

\section{Numerical Experiments}

In this section, we verify effectiveness of the explicit MONTE procedure, comparing with the AOS method (7). Both algorithms incorporates the same spatial schemes in Section 2.3 and the same constraint parameter in Section 3.1. The explicit MONTE procedure utilizes the variable timestep size (20), while the AOS method is provided with the constant timestep size which experimentally results in the best PSNR among all constant timestep sizes. A personal computer of $2.66 \mathrm{GHz}$ Celeron processor is utilized for the computation; the elapsed time (Etime) is the real time in second.

The input images are scaled by $1 / 255$ to have values between 0 and 1 . Most algorithm parameters are chosen heuristically for the algorithms to perform their best. We set $\varepsilon=0.01$ in (9); the iterations are stopped when

$$
\max _{i j}\left|u_{i j}^{n}-u_{i j}^{n-1}\right| \leq 0.01 .
$$

The ITV model [6] is selected for the numerical experiment. For simplicity and a fair comparison, we have utilized the true value of noise variance $\sigma^{2}$ for the parameter $\eta_{1}$ in (14):

$$
\eta_{1}=\frac{0.4}{\sigma^{2}} .
$$



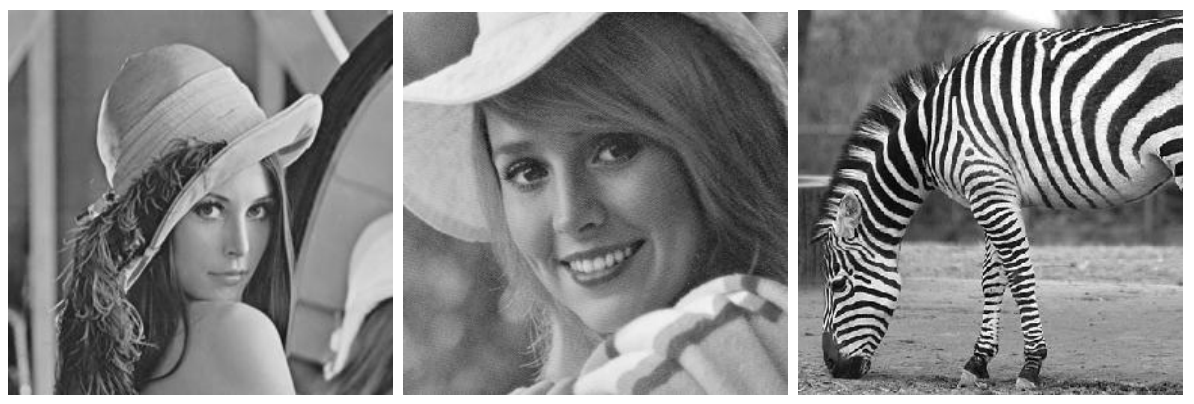

Fig. 1. Sample images: Lenna, Elaine, and Zebra.

Table 1. A PSNR analysis: comparison between the AOS and the explicit MONTE.

\begin{tabular}{|c|c||cccc|ccc|}
\hline \multicolumn{2}{|c||}{ Noise } & \multicolumn{4}{c|}{ AOS } & \multicolumn{3}{c|}{ Explicit MONTE } \\
\hline Image & PSNR & $\Delta t$ & Iter PSNR Etime & Iter PSNR Etime \\
\hline \hline Lenna & 27.27 & 0.44 & 6 & 32.14 & 0.47 & 4 & 32.83 & 0.32 \\
& 21.25 & 0.41 & 12 & 28.83 & 0.92 & 7 & 29.48 & 0.56 \\
& 16.81 & 0.42 & 15 & 26.22 & 1.20 & 9 & 26.95 & 0.72 \\
\hline Elaine & 27.28 & 0.43 & 7 & 31.51 & 0.53 & 4 & 31.91 & 0.40 \\
& 16.82 & 0.41 & 19 & 27.34 & 1.40 & 9 & 27.50 & 0.89 \\
\hline Zebra & 24.78 & 0.15 & 7 & 28.03 & 0.57 & 6 & 28.06 & 0.67 \\
& 16.82 & 0.15 & 15 & 23.01 & 1.28 & 11 & 23.34 & 1.22 \\
\hline
\end{tabular}

This choice of the parameter has been verified to be most effective (among all constant $\eta_{1}$ ) for both algorithms. See [18] for an effective estimation of $\sigma_{\mathbf{x}}^{2}$.

The algorithms have been tested various synthetic and natural images. Here we will present numerical results, obtained with the sample images in Figure 1. The peak signal-to-noise ratio (PSNR) is defined as

$$
\operatorname{PSNR} \equiv 10 \log _{10}\left(\frac{\sum_{i j} 255^{2}}{\sum_{i j}\left(g_{i j}-u_{i j}\right)^{2}}\right) \mathrm{dB}
$$

where $g$ is the original image (as in Figure 1) and $u$ denotes the restored image from a noisy image of $g$.

Table 1 contains a PSNR analysis, comparing performances of the AOS and explicit procedures applied to the sample images. The variable $\Delta t$ is the best constant timestep size we have experimentally found for the AOS, which tends to become small for texture images such as Zebra. As one can see from the table, the explicit MONTE can restore images better than the AOS method measured in PSNR for all cases. It should be noticed that the explicit scheme (17) converges in 4-11 iterations, while the AOS algorithm requires more iterations. Such a fast convergence for the explicit algorithm is due to the MONTE, the variable constraint term (14) and the variable timestep size (20), which tries to equalize the speed of diffusion over the image domain. 
(a)

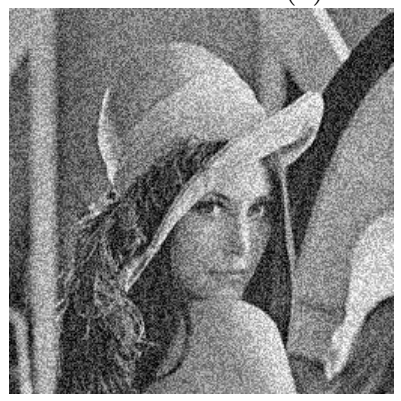

(b)

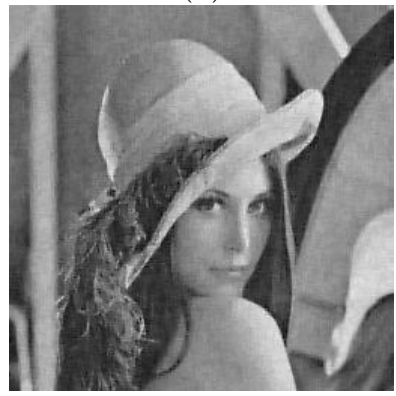

(c)

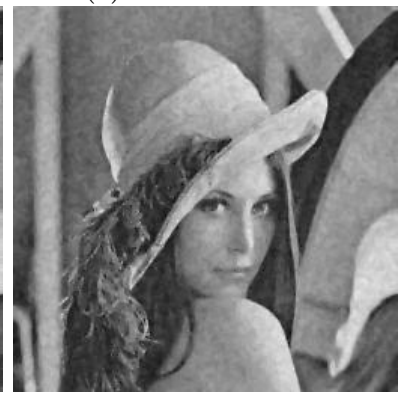

Fig. 2. Lenna image: (a) a noisy image (PSNR=21.25) and restored images by (b) the AOS method $(\mathrm{PSNR}=28.83)$ and $(\mathrm{c})$ the explicit procedure $(\mathrm{PSNR}=29.48)$.

Figure 2 depicts a noisy image of Lenna $(\mathrm{PSNR}=21.25)$ and its restored images by the two algorithms. The AOS and explicit iterations converge in 12 and 7 iterations, respectively, as shown in Table 1. As one can see from the figure, image details are preserved satisfactorily by the AOS, while the new explicit method has resulted in a better restored image. The MONTE has proved efficient in computation time and effective in the preservation of interesting image features.

The explicit MONTE procedure has converged in 4-15 iterations for all tested cases (including those not presented in this article). One should notice that the explicit procedure is efficient in computer memory, requiring only twice the image size.

\section{Conclusions}

Conventional explicit algorithms for solving PDE-based restoration models often require hundreds of iterations to converge. In this article, we have introduced strategies for spatially variable constraint parameter and timestep size, as a method of nonflat time evolution (MONTE). The explicit MONTE has been compared with the additive operator splitting (AOS) method to prove its efficiency and effectiveness. It has been numerically verified that the explicit MONTE procedure converges in only 4-15 iterations for all tested cases of image denoising. The new explicit method has converged faster and produced better restored images (measured in PSNR) than the AOS algorithm, for most cases. Since the explicit MONTE procedure is efficient in computer memory, requiring only twice the image size, it can be applied particularly for huge data sets (e.g., $3 \mathrm{D}$ images) with a great efficiency in computer memory.

\section{Acknowledgment}

The work of S. Kim is supported in part by NSF grants DMS-0312223 and DMS-0609815. 


\section{References}

1. Perona, P., Malik, J.: Scale-space and edge detection using anisotropic diffusion. IEEE Trans. on Pattern Anal. Mach. Intell. 12 (1990) 629-639

2. Alvarez, L., Lions, P., Morel, M.: Image selective smoothing and edge detection by nonlinear diffusion. II. SIAM J. Numer. Anal. 29 (1992) 845-866

3. Catte, F., Lions, P., Morel, M., Coll, T.: Image selective smoothing and edge detection by nonlinear diffusion. SIAM J. Numer. Anal. 29 (1992) 182-193

4. Kim, S.: PDE-based image restoration: A hybrid model and color image denoising. IEEE Trans. Image Processing 15 (2006) 1163-1170

5. Kim, S., Lim, H.: A non-convex diffusion model for simultaneous image denoising and edge enhancement. Electronic Journal of Differential Equations (2006) (accepted).

6. Marquina, A., Osher, S.: Explicit algorithms for a new time dependent model based on level set motion for nonlinear deblurring and noise removal. SIAM J. Sci. Comput. 22 (2000) 387-405

7. Nitzberg, M., Shiota, T.: Nonlinear image filtering with edge and corner enhancement. IEEE Trans. on Pattern Anal. Mach. Intell. 14 (1992) 826-833

8. Rudin, L., Osher, S., Fatemi, E.: Nonlinear total variation based noise removal algorithms. Physica D 60 (1992) 259-268

9. You, Y.L., Xu, W., Tannenbaum, A., Kaveh, M.: Behavioral analysis of anisotropic diffusion in image processing. IEEE Trans. Image Process. 5 (1996) 1539-1553

10. Chan, T., Shen, J.: Image Processing and Analysis. SIAM, Philadelphia (2005)

11. Osher, S., Fedkiw, R.: Level Set Methods and Dynamic Implicit Surfaces. SpringerVerlag, New York (2003)

12. Sapiro, G.: Geometric partial differential equations and image analysis. Cambridge University Press, Cambridge (2001)

13. Weickert, J., ter Haar Romeny, B., Viergever, M.: Efficient and reliable schemes for nonlinear diffusion filtering. IEEE Trans. on Image Processing 7 (1998) 398-410

14. Douglas, Jr., J., Gunn, J.: A general formulation of alternating direction methods Part I. Parabolic and hyperbolic problems. Numer. Math. 6 (1964) 428-453

15. Douglas, Jr., J., Kim, S.: Improved accuracy for locally one-dimensional methods for parabolic equations. Mathematical Models and Methods in Applied Sciences 11 (2001) 1563-1579

16. Cha, Y., Kim, S.: Edge-forming methods for image zooming. J. Mathematical Imaging and Vision (2006) (in press).

17. Cha, Y., Kim, S.: Edge-forming methods for color image zooming. IEEE Trans. Image Process. (2006) (in press).

18. Kim, S., Kwon, S.H.: Efficiency and reliability in nonlinear diffusion filtering. (in preparation) 